\title{
Prevalence of Neuropsychiatric Symptoms across the Declining Memory Continuum: An Observational Study in a Memory Clinic Setting
}

\author{
Meiyan Zhang Huali Wang Tao Li Xin Yu \\ Dementia Care and Research Center, Peking University Institute of Mental Health, and Key \\ Laboratory for Mental Health, Ministry of Health, Beijing, China
}

\section{Key Words}

Alzheimer's disease • Mild cognitive impairment • Subjective cognitive impairment •

Neuropsychiatric symptoms $\cdot$ Prevalence

\begin{abstract}
Aims: The study aimed to compare the frequency of neuropsychiatric symptoms (NPS) across the declining memory continuum, from normal aging, subjective cognitive impairment (SCl), and mild cognitive impairment (MCl) to Alzheimer's disease (AD), and to explore the clinical correlates of NPS. Method: In a memory clinic, 157 subjects (46 mild AD patients, $38 \mathrm{MCl}$ individuals, $24 \mathrm{SCl}$ subjects, and 49 normal controls) completed the neurobehavioral assessments with the Neuropsychiatric Inventory (NPI). The clinical significance of each NPI domain was defined as an item score $\geq 4$. Result: Clinically significant depression was more common in the $\mathrm{SCl}$ than in the normal control group $(p<0.05)$. The frequency of NPS was significantly greater in the mild AD group compared to other groups. Clinically significant apathy and aberrant motor behavior were more common among the AD group than the MCl group $(p<0.05)$. The MMSE score (OR $4.84,95 \% \mathrm{Cl} 1.92-12.16, \mathrm{p}=0.001)$ and apathy (OR 12.73, 95\% Cl 1.48-109.68, $\mathrm{p}=0.021)$ significantly determined the diagnostic status as $\mathrm{MCl}$ or mild AD. Conclusion: Across the declining memory continuum, the frequency of NPS was highest among mild AD patients. Depression, apathy, and aberrant motor behavior deserve more attention. Presence of apathy might be an independent determinant for mild AD.

Copyright $\odot 2012$ S. Karger AG, Basel
\end{abstract}

Part of this paper has been presented at the 3rd Annual Meeting of the Asian Society against Dementia in October 11-13, 2009, Seoul, Korea. 


\section{Background}

For patients with dementia, the presence of neuropsychiatric symptoms (NPS) usually results in institutionalization, placement in nursing homes, increased utilization of medical resources, more caregiver stress, diminished quality of life for the persons with dementia and their families, and greater economic burden on the caregivers [1]. A preliminary survey in China had demonstrated that economic burden of dementia was significantly correlated with the presence of NPS [2].

Over the disease course of dementia, $80-90 \%$ of patients with dementia experienced NPS [3]. In the Cardiovascular Health Study, 75\% of dementia patients exhibited NPS, $62 \%$ were rated as clinically significant, and the most common disturbances were apathy, depression, and agitation [4]. A research conducted in nursing homes and neurology outpatient departments in the northern part of Taiwan showed that $100 \%$ of dementia patients had at least one NPS. The most common NPS in order of decreasing frequency were nighttime behavior, apathy, aberrant motor behavior (AMB), and depression among dementia patients. In the outpatient department, nighttime behavior, apathy, $\mathrm{AMB}$, euphoria, and depression were common [5].

Mild cognitive impairment (MCI) has emerged as an identifiable condition and, in many cases, a likely state preceding diagnosable Alzheimer's disease (AD) [6]. There is an annual conversion rate of $10-15 \%$ from amnesic-type MCI to AD [7, 8]. NPS were common in MCI: $43 \%$ of MCI participants exhibited NPS, $29 \%$ were rated as clinically significant, depression, apathy, and irritability being the most common [4]. In a systematic review, it was found that the prevalence rate of NPS in MCI ranged from 35 to 85\%. Depression, anxiety, and irritability were most common. NPS, particularly depression, might represent risk factors for MCI or predictors for the conversion of MCI to AD [9].

Subjective cognitive impairment (SCI) is also called subjective memory complaints. Some researchers found that SCI was common in old people. The prevalence varied from 22 to $50 \%$. SCI was associated with aging, female gender, low level of education, depression, and lower MMSE scores [10,11]. SCI was a stage prior to MCI in the eventual development of AD dementia [12]. Reisberg et al. [13] found that (1) there was support from at least one longitudinal study for a long-standing concept of SCI as a pre-MCI condition lasting approximately 15 years; (2) there were complex relationships between SCI and depression and anxiety; (3) differences between SCI subjects and age-matched non-SCI persons were being published in terms of cognitive tests, hippocampal gray matter density, hippocampal volumes, cerebral metabolism, and urinary cortisol levels. It was one of the few reports about the NPS of SCI to date.

Although there have been many reports on NPS of MCI and dementia, currently, few studies compare these disturbances across the declining memory continuum, from normal cognition, SCI, and MCI to AD. Therefore, we tested the hypothesis that, as cognitive impairment progresses from normal cognition, SCI, and MCI to AD, the prevalence of NPS would increase. In addition, we also proposed to explore the clinical correlates of NPS among individuals with $\mathrm{MCI}$ and mild AD.

\section{Methods}

All subjects who had completed the neuropsychological and neurobehavioral assessments were retrieved from the database of the Dementia Care and Research Center, Peking University Institute of Mental Health, from March, 2006, to March, 2010. A total of 157 subjects (46 mild AD patients, $38 \mathrm{MCI}$ individuals, 24 SCI subjects, and 49 normal controls, NC) were included in this analysis. 


\section{Inclusion Criteria}

The diagnosis of AD was made according to NINCDS-ADRDA criteria [14]. Individuals with MCI met Petersen criteria including (1) memory complaint (preferably corroborated by a caregiver); (2) normal activities of daily living; (3) normal general cognitive function; (4) abnormal memory on cognitive testing (generally performance $\geq 1.5$ SD below age and education-adjusted scores), and (5) the absence of dementia (clinical judgment) [6]. SCI was defined by the presence of memory complaints $\geq 6$ months, normal objective memory performance, and normal activities of daily living. NC was defined by absence of memory complaints, normal objective memory performance, and normal activities of daily living [15].

\section{Instruments}

The Neuropsychiatric Inventory (NPI) was the major instrument for the assessment of NPS [16]. The Chinese version of the NPI has been validated in Chinese communities $[17,18]$ and was used in this study to assess NPS, including domains of delusions, hallucinations, agitation/aggression, depression/dysphoria, anxiety, euphoria/elation, apathy/indifference, disinhibition, irritability/lability, AMB, nighttime behavior disturbance, and changes in appetite and eating behavior. Severity and frequency of these domains were evaluated based on an interview with an informant, primarily family members, by a trained rater. The score of each domain was calculated by multiplying the frequency score by the severity score, and the sum of 12 domain scores constituted the total NPI score. Presence of individual NPS was defined as the domain score $>0$. The presence of clinically significant NPS was defined as the domain score $\geq 4$.

The Chinese version of the Mini-Mental State Examination (MMSE) was used to assess the general cognitive function of all subjects [19]. Mild AD was defined as having a total MMSE score $>20[20]$.

\section{Analysis}

Statistical analysis was performed with the Statistical Package for Social Sciences, version 16 (SPSS Inc., Chicago, Ill., USA). The frequency of clinically significant NPS among the four groups was compared with the Kruskal-Wallis $H$ test. A $2 \times 2$ crosstabulation $\chi^{2}$ test was used for comparisons of categorical data between the two groups. For the $2 \times 2$ crosstabulation $\chi^{2}$ test, Fisher's exact test was deployed if there were one or more cell(s) having an expected count $<5$. Statistical significance was set at a $\mathrm{p}$ value $<0.05$ throughout.

The association between scores of each NPS domain and cognitive assessment scores (i.e., MMSE) was assessed by partial correlation while controlling for age and educational levels. To identify the effects of cognitive function and presence of clinically significant NPS on determining subject status, i.e., $\mathrm{AD}, \mathrm{MCI}$, and cognitively normal, the Hosmer-Lemeshow test was first deployed and found that the variables were fit for logistic regression analysis for $\mathrm{AD}$ against $\mathrm{MCI}$ or cognitively normal groups. Hence, multivariate logistic regression analyses were conducted. Age, educational level, gender, and MMSE score were included throughout the analyses. For each analysis, only clinically significant NPS bearing statistical significance between the two groups were included. Age, education, and MMSE score were included as continuous variables, and gender and presence of NPS were considered as categorical variables. The reference category was female, and presence of clinically significant NPS. The variables to determine the status of MCI against normal cognition were not fit for logistic regression analysis; the multivariate discriminant analysis, specifically with Wilks' $\lambda$ stepwise method and leave-one-out classification was introduced. Throughout the multivariate regression analysis and discriminant analysis, variables were entered with the probability of $\mathrm{F}<0.05$ and were removed at $\mathrm{p}>0.10$. 
Zhang et al.: Prevalence of NPS across the Declining Memory Continuum

Table 1. Demographic and clinical characteristics of study participants

\begin{tabular}{lllllrr}
\hline Characteristic & $\begin{array}{l}\text { Mild AD } \\
(\mathrm{n}=46)\end{array}$ & $\begin{array}{l}\text { MCI } \\
(\mathrm{n}=38)\end{array}$ & $\begin{array}{l}\text { SCI } \\
(\mathrm{n}=24)\end{array}$ & $\begin{array}{l}\mathrm{NC} \\
(\mathrm{n}=49)\end{array}$ & $\mathrm{F}\left(\chi^{2}\right)$ & $\mathrm{p}$ \\
\hline Gender (male/female) & $17 / 29$ & $21 / 17$ & $8 / 16$ & $27 / 22$ & 5.99 & 0.112 \\
Age, years & $75 \pm 6$ & $71 \pm 8$ & $71 \pm 8$ & $70 \pm 9$ & 4.77 & 0.003 \\
Married, \% & 73.9 & 94.7 & 75.0 & 87.8 & 19.57 & 0.026 \\
Education, years & $13 \pm 5$ & $14 \pm 3$ & $15 \pm 3$ & $14 \pm 3$ & 1.63 & 0.186 \\
MMSE score & $23 \pm 2$ & $28 \pm 1$ & $29 \pm 1$ & $29 \pm 1$ & 137.25 & $<0.001$ \\
\hline
\end{tabular}

Data are mean \pm SD unless otherwise indicated.

\section{Results}

\section{Demographic and Clinical Characteristics of the Four Groups}

The four groups were well matched for gender and education. Compared to the MCI and NC groups, age was significantly higher in the mild AD group $(\mathrm{p}<0.05)$. Marital status was significantly different between the MCI group and the SCI group $(\mathrm{p}<0.05)$. The mean MMSE score was lower and ADL score was greater in the mild AD group than in the other three groups $(p<0.05)$, indicating that cognitive function and abilities of daily living were worst in the mild AD group. The MMSE score was lower in the MCI group than in the SCI and NC groups (table 1).

\section{Frequency of NPS among the Four Groups}

Among mild AD patients, $95.7 \%$ had at least one symptom, which was a significantly higher percentage than in the other three groups $(\mathrm{p}<0.05)$. In the mild AD group, the most common symptoms were apathy (87.0\%), irritability (54.3\%), agitation (34.8\%), anxiety (32.6\%), and depression (30.4\%). In the MCI group, $81.6 \%$ had at least one symptom and the most common symptoms were irritability (44.7\%), apathy (39.5\%), depression (28.9\%), sleep disturbance (26.3\%), and anxiety (26.3\%). In the SCI group, $79.2 \%$ had at least one symptom and the most common symptoms were irritability (54.2\%), apathy (33.3\%), and depression (29.2\%). When comparing the four groups, the frequency of depression was higher in the $\mathrm{MCI}$ and SCI groups than in the NC group $(\mathrm{p}<0.05)$. In the mild AD group, agitation, apathy, and AMB were significantly more common than in the other three groups $(\mathrm{p}<0.05)$. The mild AD group also had more frequently delusion than the SCI group and more delusion, depression, and anxiety than the NC group ( $p<0.05$; table 2$)$.

Concerning the clinically significant NPS, the most common symptoms were nighttime behaviors (10.2\%), apathy/indifference (6.1\%), anxiety (4.1\%), and irritability/lability (4.1\%) in the NC group, nighttime behavior (20.8\%), apathy/indifference (20.8\%), and depression/ dysphoria (8.3\%) in the SCI group, nighttime behaviors (21.1\%), apathy/indifference (18.4\%), irritability/lability (15.8\%), and anxiety (15.8\%) in the MCI group, and apathy/indifference (80.4\%), irritability/lability (30.4\%), and AMB (23.9\%) in the mild AD group (fig. 1). Group comparisons showed that there were significant differences in the frequency of delusion $\left(\chi^{2}=15.21, \mathrm{p}=0.002\right)$, agitation $\left(\chi^{2}=11.14, \mathrm{p}=0.011\right)$, apathy $\left(\chi^{2}=67.52, \mathrm{p}=0.000\right)$, irritability/liability $\left(\chi^{2}=15.59, \mathrm{p}=0.001\right)$, and AMB $\left(\chi^{2}=21.71, \mathrm{p}=0.000\right)$ among the four groups. The frequency of depression/dysphoria $\left(\chi^{2}=6.76, p=0.080\right)$ and anxiety $\left(\chi^{2}=6.39, p=\right.$ 0.094 ) showed a trend to differ among the four groups. Post-hoc comparisons showed that there was no significant difference in the frequency of clinically significant NPS between the 
Table 2. Prevalence (\%) of NPS (NPI domain score $>0$ ) in mild AD, MCI, SCI, and NC groups

\begin{tabular}{|c|c|c|c|c|c|c|c|c|c|c|c|}
\hline \multirow[t]{2}{*}{ NPI domain } & \multirow{2}{*}{$\begin{array}{l}\text { Mild AD } \\
(n=46)\end{array}$} & \multirow{2}{*}{$\begin{array}{l}\text { MCI } \\
(\mathrm{n}=38)\end{array}$} & \multirow{2}{*}{$\begin{array}{l}\text { SCI } \\
(n=24)\end{array}$} & \multirow{2}{*}{$\begin{array}{l}\mathrm{NC} \\
(\mathrm{n}=49)\end{array}$} & \multirow{2}{*}{$\begin{array}{l}\mathrm{K}-\mathrm{W} \\
\text { test } \\
\mathrm{p}\end{array}$} & \multicolumn{6}{|c|}{$\mathrm{p}$ for post-hoc comparison } \\
\hline & & & & & & $\begin{array}{l}\text { SCI vs. } \\
\text { NC }\end{array}$ & $\begin{array}{l}\text { MCI vs. } \\
\text { NC }\end{array}$ & $\begin{array}{l}\text { AD vs. } \\
\text { NC }\end{array}$ & $\begin{array}{l}\text { MCI vs. } \\
\text { SCI }\end{array}$ & $\begin{array}{l}\text { AD vs. } \\
\text { SCI }\end{array}$ & $\begin{array}{l}\text { AD vs. } \\
\text { MCI }\end{array}$ \\
\hline Delusion & 23.9 & 10.5 & 0 & 4.1 & 0.002 & n.s. & n.s. & 0.01 & n.s. & 0.01 & n.s. \\
\hline Hallucination & 6.5 & 2.6 & 4.2 & 2.0 & n.s. & n.s. & n.s. & n.s. & n.s. & n.s. & n.s. \\
\hline Agitation/aggression & 34.8 & 13.2 & 8.3 & 12.2 & 0.008 & n.s. & n.s. & 0.01 & n.s. & 0.02 & 0.02 \\
\hline Depression/dysphoria & 30.4 & 28.9 & 29.2 & 8.2 & 0.032 & 0.02 & 0.01 & 0.01 & n.s. & n.s. & n.s. \\
\hline Anxiety & 32.6 & 26.3 & 12.5 & 14.3 & n.s. & n.s. & n.s. & 0.04 & n.s. & n.s. & n.s. \\
\hline Euphoria & 2.2 & 0 & 0 & 0 & n.s. & n.s. & n.s. & n.s. & n.s. & n.s. & n.s. \\
\hline Apathy/indifference & 87.0 & 39.5 & 33.3 & 22.4 & $<0.001$ & n.s. & n.s. & $<0.001$ & n.s. & $<0.001$ & $<0.001$ \\
\hline Disinhibition & 13.0 & 2.6 & 4.2 & 4.1 & n.s. & n.s. & n.s. & n.s. & n.s. & n.s. & n.s. \\
\hline Irritability/lability & 54.3 & 44.7 & 54.2 & 38.8 & n.s. & n.s. & n.s. & n.s. & n.s. & n.s. & n.s. \\
\hline $\mathrm{AMB}$ & 28.3 & 5.3 & 0 & 0 & $<0.001$ & n.s. & n.s. & $<0.001$ & n.s. & 0.01 & 0.01 \\
\hline Nighttime behaviors & 19.6 & 26.3 & 25.0 & 20.4 & n.s. & n.s. & n.s. & n.s. & n.s. & n.s. & n.s. \\
\hline Appetite/eating change & 6.5 & 5.3 & 4.2 & 0 & n.s. & n.s. & n.s. & n.s. & n.s. & n.s. & n.s. \\
\hline NPI & 95.7 & 81.6 & 79.2 & 63.3 & 0.001 & n.s. & n.s. & $<0.001$ & n.s. & 0.03 & 0.04 \\
\hline
\end{tabular}

K-W test = Kruskal-Wallis test; n.s. = no significance.

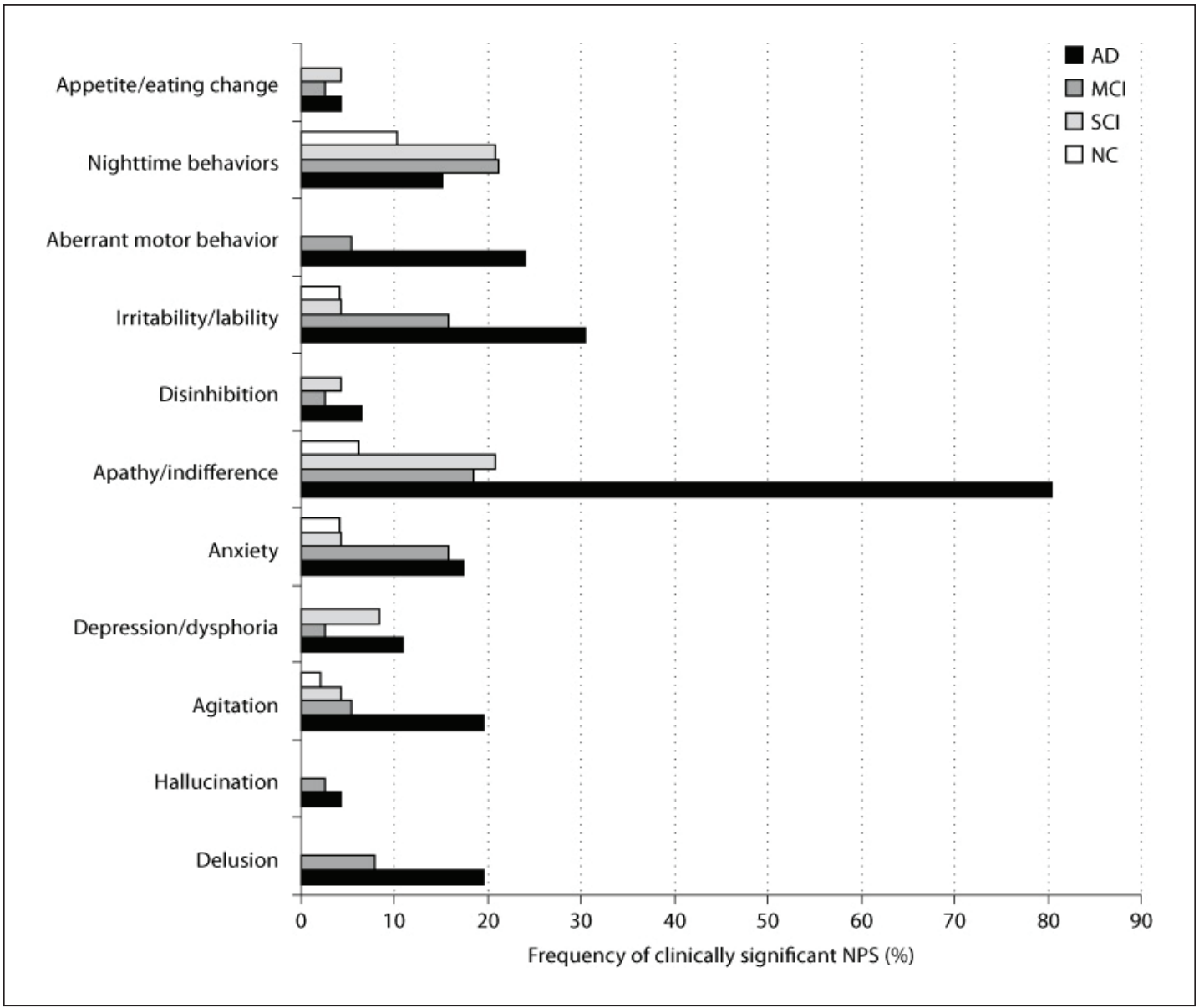

Fig. 1. Frequency of clinically significant NPS (NPI domain score $\geq 4$ ) in mild AD, MCI, SCI, and NC groups. 
Table 3. Logistic regression for clinical correlates of NC, MCI, and $\mathrm{AD}$ groups

Zhang et al.: Prevalence of NPS across the Declining Memory Continuum

\begin{tabular}{lrlrl}
\hline & Wald & p & OR & $95 \%$ CI \\
\hline Cognitively normal vs. AD & & & & \\
$\quad$ Gender & 3.70 & 0.055 & 33.34 & $0.93-0.001$ \\
MMSE score & 7.94 & 0.005 & 6.40 & $1.76-23.28$ \\
Apathy score & 5.19 & 0.023 & 21.82 & $1.54-309.71$ \\
MCI vs. AD & & & & \\
$\quad$ MMSE score & 11.23 & 0.001 & 4.84 & $1.92-12.16$ \\
Apathy score & 5.36 & 0.021 & 12.73 & $1.48-109.68$ \\
\hline
\end{tabular}

SCI and NC groups ( $p>0.05)$. Therefore, the subjects in the SCI group were combined with NCs as cognitively normal. The frequency of delusion (Fisher's exact $\mathrm{p}=0.038$ ) was significantly different between the MCI and cognitively normal groups. The frequency of delusion (Fisher's exact $\mathrm{p}=0.000$ ), agitation (Fisher's exact $\mathrm{p}=0.003$ ), anxiety (Fisher's exact $\mathrm{p}=$ $0.022)$, apathy $\left(\chi^{2}=57.92, p=0.000\right)$, irritability $\left(\chi^{2}=15.97, p=0.000\right)$, and AMB (Fisher's exact $p=0.000)$ was significantly greater in the AD group relative to cognitively normal subjects. The frequency of apathy $\left(\chi^{2}=32.08, \mathrm{p}=0.000\right)$ and $\mathrm{AMB}\left(\chi^{2}=5.53, \mathrm{p}=0.019\right)$ was significantly higher in the AD group compared with the MCI group.

\section{Clinical Correlates of NPS}

After controlling for effects of age and education, mean scores for hallucination (partial correlation, $\mathrm{r}=-0.337$, d.f. $=42, \mathrm{p}=0.025$ ) and appetite/eating change (partial correlation, $\mathrm{r}=-0.304$, d.f. $=42, \mathrm{p}=0.045$ ) were correlated with mean MMSE score in the AD group. In the MCI group, the mean apathy score was associated with the mean MMSE score (partial correlation, $\mathrm{r}=-0.379$, d.f. $=34, \mathrm{p}=0.023$ ). There was no significant correlation between the subscores of NPS and MMSE scores in the cognitively normal group. Logistic regression analysis showed that MMSE score (OR 6.40, 95\% CI 1.76-23.28, $\mathrm{p}=0.005)$ and presence of apathy (OR 21.82, 95\% CI 1.54-309.71, $\mathrm{p}=0.023)$ were significant determinants for subjects' group status as cognitively normal or mild AD (Hosmer-Lemeshow test $\chi^{2}=0.935$, d.f. $=8$, $\mathrm{p}=0.999, \mathrm{R}^{2}=0.69,-2 \log$ likelihood $\left.=18.27\right)$. MMSE score (OR 4.84, 95\% CI 1.92-12.16, $\mathrm{p}=0.001)$ and apathy score (OR $12.73,95 \%$ CI $1.48-109.68, \mathrm{p}=0.021)$ also significantly determined the diagnostic status as MCI or mild AD (Hosmer-Lemeshow test $\chi^{2}=1.408$, d.f. $=8, p=0.994, R^{2}=0.65,-2 \log$ likelihood $=26.80$; table 3). Stepwise linear regression analysis showed that MMSE score solely contributed as a determinant of group status as MCI or cognitively normal (Wilks' $\lambda=0.86, \mathrm{~F}=17.58, \mathrm{p}=0.000$, cross-validated classification accuracy $=70.3 \%)$.

\section{Discussion}

This is the first study in mainland China to systematically investigate and compare the prevalence of NPS across the declining memory continuum in a memory clinic setting. In the present study, the frequency and presentation of NPS in mild AD were significantly different from MCI, SCI, and NC. There were $95.7 \%$ of mild AD patients who had at least one symptom. The most common symptoms were apathy, irritability, agitation, anxiety, and depression. Our findings confirm previous estimates of a high prevalence rate and a common presentation of NPS in dementia $[21,22]$. Many studies reported that the most common NPS in patients with mild AD were apathy, depression, anxiety, and irritability [23- 
26]. In our study, we also found that the frequency of clinically significant NPS was high in mild AD. The most common clinically significant symptoms were apathy, irritability, and $A M B$. The frequencies of apathy and AMB were significantly greater than in the other three groups.

The frequency of NPS in the MCI group was $81.6 \%$. The most common symptoms were irritability, apathy, depression, sleep, and anxiety. The results were similar as in previous studies. In the Cardiovascular Health Study, 43\% of MCI patients exhibited NPS, with depression, apathy, and irritability being the most common symptoms [4]. Hwang et al. [27] found that the most common symptoms in MCI were depression, apathy, irritability, and anxiety. Rozzini et al. [28] also found that about $85 \%$ of MCI patients had some NPS, and the most prevalent symptom was depression, followed by anxiety. In a review by Monastero et al. [9], the global prevalence of NPS in MCI ranged from 35 to 85\%, the most common behavioral symptoms were depression, anxiety, and irritability, and hospital-based samples reported a higher global prevalence of NPS than population-based studies. Our study also found that the most common clinically significant symptoms were sleep and apathy in the MCI group. Although NPS were common in the MCI group, clinically significant NPS were relatively rare.

SCI was common in aged persons: about $22-50 \%$ of $>65$-year-old persons reported SCI in the community $[10,11,29]$. But there has been little research on NPS associated with SCI. In the present study, $79.2 \%$ of the SCI group had at least one symptom. The most common symptoms were irritability, apathy, and depression. In the SCI group, the most common clinically significant symptoms were sleep and apathy. Clinically significant NPS were relatively rare in the SCI group.

The frequency of NPS, in particular agitation, apathy, and AMB, was significantly greater among mild AD patients than in the other three groups. Clinically significant symptoms, such as apathy and AMB, were also more common in mild AD than in MCI, SCI, and NC. There were significant associations between cerebral white-matter changes and apathy [30]. Brain white-matter hyperintensities were associated with symptoms of AMB [31]. It was suggested that brain structure changes may be associated with the presence of NPS in AD. In the stage of mild $\mathrm{AD}$, the brain structure might have changed so that the NPS in mild AD were different from MCI, SCI, and NC.

In the present study, the frequency of depression was greater in MCI and SCI than in NC. Modrego et al. [32] found that patients with MCI and depression had more than twice the risk of developing AD than those without depression. Gabryelewicz et al. [33] observed that the occurrence of depressive symptoms might constitute a predictor for those who are more likely to progress to dementia. According to Teng et al. [34], depression and apathy appear to be the most useful symptoms to identify MCI subjects at a higher risk of developing dementia. Subjects with MCI might have neuropathological changes of AD, and the abnormalities were most severe in the limbic system $[35,36]$. Thus, the mood disturbances in MCI might reflect the pathologic and neurochemical changes of the limbic system.

We found that apathy may be useful in identifying individuals with mild AD and distinguishing mild AD from MCI. Apathy has been found to discriminate between subjects with normal cognition and subjects with MCI in a Chinese community [37]. The finding is of great clinical significance. In Chinese culture, caregivers may not easily identify apathy as it is less likely to cause destructive behavior [38]. To better identify individuals with suspected dementia, clinicians should be trained to recognize NPS, in particular apathy, in the clinical practice.

There are several limitations of this study. The subjects were referred from a memory clinic setting; therefore, the sample may not be representative of community samples of individuals with mild AD, MCI, and SCI. Furthermore, the assessment was cross-sectional and 
was unable to examine the causal link between the clinical correlates and NPS. Thus, longitudinal follow-up studies are required to delineate these relationships.

In summary, across the declining memory continuum, the frequency of NPS was the highest among mild AD patients. The NPS in AD were significantly different from those in MCI, SCI, and NC. NPS were prevalent among individuals with SCI and MCI, though without reaching clinical significance. The symptoms of depression, apathy, and AMB deserve more attention in the declining memory continuum. Apathy might be a predictor of the progression from cognitively normal to $\mathrm{MCI}$ and eventually to AD.

\section{Acknowledgements}

This study was supported by the Natural Science Foundation of China (30500178, 30971044, 81171018), Hi-tech R\&D Program, Ministry of Science and Technology (2007AA02Z421), and the National Institute on Aging and Fogarty International Center (R21AG028180-01A2). The authors thank Dr. Wai-Chi Chan for kind comments and editorial assistance.

\section{References}

-1 Edell WS, Tunis SL: Antipsychotic treatment of behavioral and psychological symptoms of dementia in geropsychiatric inpatients. Am J Geriatr Psychiatry 2001;9:289-297.

2 An CX, Yu X: Economic burden and related factors in patients with dementia. Chinese Mental Health Journal 2005;19:592-594.

-3 Steinberg M, Tschanz JT, Corcoran C, et al: The persistence of neuropsychiatric symptoms in dementia: the Cache County Study. Int J Geriatr Psychiatry 2004;19:19-26.

-4 Lyketsos CG, Lopez O, Jones B, Fitzpatrick AL, Breitner J, DeKosky S: Prevalence of neuropsychiatric symptoms in dementia and mild cognitive impairment: results from the cardiovascular health study. JAMA 2002;288:1475-1483.

5 Hsieh CJ, Chang CC, Lin CC: Neuropsychiatric profiles of patients with Alzheimer's disease and vascular dementia in Taiwan. Int J Geriatr Psychiatry 2009;24:570-577.

-6 Petersen RC, Smith GE, Waring SC, Ivnik RJ, Tangalos EG, Kokmen E: Mild cognitive impairment: clinical characterization and outcome. Arch Neurol 1999;56:303-308.

-7 Petersen RC, Doody R, Kurz A, et al: Current concepts in mild cognitive impairment. Arch Neurol 2001;58:1985-1992.

8 Tierney MC, Szalai JP, Snow WG, et al: Prediction of probable Alzheimer's disease in memory-impaired patients: a prospective longitudinal study. Neurology 1996;46:661-665.

-9 Monastero R, Mangialasche F, Camarda C, Ercolani S, Camarda R: A systematic review of neuropsychiatric symptoms in mild cognitive impairment. J Alzheimers Dis 2009;18:11-30.

-10 Jonker C, Geerlings MI, Schmand B: Are memory complaints predictive for dementia? A review of clinical and population-based studies. Int J Geriatr Psychiatry 2000;15:983-991.

-11 Kim JM, Stewart R, Shin IS, Choi SK, Yoon JS: Subjective memory impairment, cognitive function and depression - a community study in older Koreans. Dement Geriatr Cogn Disord 2003;15:218 225.

12 Reisberg B, Gauthier S: Current evidence for subjective cognitive impairment (SCI) as the pre-mild cognitive impairment (MCI) stage of subsequently manifest Alzheimer's disease. Int Psychogeriatr 2008;20:1-16.

-13 Reisberg B, Prichep L, Mosconi L, et al: The pre-mild cognitive impairment, subjective cognitive impairment stage of Alzheimer's disease. Alzheimers Dement 2008;4:S98-S108.

14 McKhann G, Drachman D, Folstein M, Katzman R, Price D, Stadlan EM: Clinical diagnosis of Alzheimer's disease: report of the NINCDS-ADRDA Work Group under the auspices of Department of Health and Human Services Task Force on Alzheimer's Disease. Neurology 1984;34:939-944. 
15 He Y, Qu G, Xiong X: The assessment of activities of daily living for the elderly. Shanghai Archive of Psychiatry 1989;1:124-126.

-16 Cummings JL, Mega M, Gray K, Rosenberg-Thompson S, Carusi DA, Gornbein J: The Neuropsychiatric Inventory: comprehensive assessment of psychopathology in dementia. Neurology 1994;44: 2308-2314.

17 Xie H, Wang L, Yu X, et al: Neuropsychiatric symptoms in dementia and elderly people in the community: results from the Beijing Dementia Cooperative Study. Chin J Epidemiol 2004;25:829-832.

18 Xie H, Wang L, Yu X, et al: A factor analysis of neuropsychiatric syndromes in dementia: results from the Beijing Dementia Cooperative Study. Chin J Psychiatry 2005;38:206-209.

19 Li G, Shen Y, Chen C, et al: Preliminary study on Chinese version of Mini-Mental Status Examination. Chinese Mental Health Journal 1989;4:148-151.

-20 Forchetti CM: Treating patients with moderate to severe Alzheimer's disease: implications of recent pharmacologic studies. Prim Care Companion J Clin Psychiatry 2005;7:155-161.

-21 Cheng TW, Chen TF, Yip PK, Hua MS, Yang CC, Chiu MJ: Comparison of behavioral and psychological symptoms of Alzheimer's disease among institution residents and memory clinic outpatients. Int Psychogeriatr 2009;21:1134-1141.

-22 Suh GH, Kim SK: Behavioral and Psychological Signs and Symptoms of Dementia (BPSD) in antipsychotic-naive Alzheimer's disease patients. Int Psychogeriatr 2004;16:337-350.

-23 Piccininni M, Di Carlo A, Baldereschi M, Zaccara G, Inzitari D: Behavioral and psychological symptoms in Alzheimer's disease: frequency and relationship with duration and severity of the disease. Dement Geriatr Cogn Disord 2005;19:276-281.

24 Shih CS, Yan SH, Ho YH, Lin YT, Li JY, Lo YK: Psychiatric morbidity in dementia patients in a neurology-based memory clinic. Acta Neurol Taiwan 2005;14:179-186.

-25 Shimabukuro J, Awata S, Matsuoka H: Behavioral and psychological symptoms of dementia characteristic of mild Alzheimer patients. Psychiatry Clin Neurosci 2005;59:274-279.

-26 Yener GG: The neuropsychiatric inventory scores change across the Mini Mental State Examination ranges in patients with Alzheimer's disease: a multicenter study in Turkey. Cogn Behav Neurol 2009; 22:264-269.

-27 Hwang TJ, Masterman DL, Ortiz F, Fairbanks LA, Cummings JL: Mild cognitive impairment is associated with characteristic neuropsychiatric symptoms. Alzheimer Dis Assoc Disord 2004;18:17-21.

-28 Rozzini L, Vicini Chilovi B, Conti M, et al: Neuropsychiatric symptoms in amnestic and nonamnestic mild cognitive impairment. Dement Geriatr Cogn Disord 2008;25:32-36.

29 Gagnon M, Dartigues JF, Mazaux JM, et al: Self-reported memory complaints and memory performance in elderly French community residents: results of the PAQUID Research Program. Neuroepidemiology 1994;13:145-154.

-30 Jonsson M, Edman A, Lind K, Rolstad S, Sjögren M, Wallin A: Apathy is a prominent neuropsychiatric feature of radiological white-matter changes in patients with dementia. Int J Geriatr Psychiatry 2010;25:588-595.

-31 Berlow YA, Wells WM, Ellison JM, Sung YH, Renshaw PF, Harper DG: Neuropsychiatric correlates of white matter hyperintensities in Alzheimer's disease. Int J Geriatr Psychiatry 2010;25:780-788.

- 32 Modrego PJ, Ferrandez J: Depression in patients with mild cognitive impairment increases the risk of developing dementia of Alzheimer type: a prospective cohort study. Arch Neurol 2004;61:1290-1293.

-33 Gabryelewicz T, Styczynska M, Luczywek E, et al: The rate of conversion of mild cognitive impairment to dementia: predictive role of depression. Int J Geriatr Psychiatry 2007;22:563-567.

-34 Teng E, Lu PH, Cummings JL: Neuropsychiatric symptoms are associated with progression from mild cognitive impairment to Alzheimer's disease. Dement Geriatr Cogn Disord 2007;24:253-259.

-35 Morris JC, Storandt M, Miller JP, et al: Mild cognitive impairment represents early-stage Alzheimer disease. Arch Neurol 2001;58:397-405.

-36 Grober E, Dickson D, Sliwinski MJ, et al: Memory and mental status correlates of modified Braak staging. Neurobiol Aging 1999;20:573-579.

- 37 Chan WC, Lam LC, Tam CW, et al: Prevalence of neuropsychiatric symptoms in Chinese older persons with mild cognitive impairment - a population-based study. Am J Geriatr Psychiatry 2010;18: 948-954.

38 Ma WX, Wang HL, Cummings JL, Yu X: Reliability and validity of Chinese version of Neuropsychiatric Inventory-Questionnaire in patients with Alzheimer's disease. Chinese Mental Health Journal 2010;24:338-342. 